\title{
Wounded Healers: Coping with Vicarious Trauma among Mental Health Professionals
}

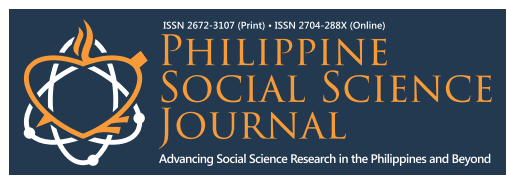

Graziela B. Salvilla ${ }^{1}$ and Chris John S. Bedoria ${ }^{2}$

${ }^{1}$ Colegio San Agustin-Bacolod, Bacolod City, Philippines

2University of the Philippines-Diliman, Quezon City, Philippines

\section{Article history:}

Submitted: 12 November 2021

Revised: 12 December 2021

Accepted: 21 December 2021

\section{Keywords:}

Psychology

Coping strategies

Vicarious trauma

Mental health professionals

Negros Occidental
ABSTRACT. Mental health professionals have been experiencing vicarious trauma due to their exposure to trauma-related cases. This study explored the coping strategies used by mental health professionals in dealing with vicarious trauma. A purposive sample of five (5) mental health professionals participated in this study. The study utilized Narrative Inquiry and Thematic Analysis to explore the stories of handling trauma cases and construct the central themes of coping strategies from vicarious trauma among mental health professionals. Findings of the study generated six central themes: a) recognition of experienced vicarious trauma symptoms, b) healthy personal and working relationships, c) fulfilling self-preserving needs, d) regulation of thought processes, e) professional competence and due care, and f) fate and faith. The results provided a holistic approach to vicarious trauma that targeted all dimensions of an individual; emotional, physiological, cognitive, occupational, and spiritual.

\subsection{Introduction}

Vicarious trauma, by definition, is a form of Post-Traumatic Stress Disorder (PTSD) that occurs when professionals become repositories for other people's feelings (Laidlaw, 2018). Specifically, a traumatic event is an incident that causes physical, emotional, spiritual, or psychological harm. As a result, the person experiencing the distressing event may feel threatened, anxious, or frightened (Cafasso, 2016). As stipulated in the Diagnostic and Statistical Manual of Mental Disorders, Fifth Edition (DSM-5), one criterion to develop PTSD includes exposure to actual or threatened death, serious injury, or sexual violence. Specifically, repeated or extreme exposure to details of the event (American Psychiatric Association [APA], 2013).

Hunt (2018) propounded that persons most susceptible to developing vicarious trauma are mental health professionals who handled trauma cases. Molnar et al. (2020) also surmised that manifestations of vicarious trauma among child welfare and child protection professionals were described into three conditions; (a) PTSD and other psychological impacts; (b) effects on the ability to work; and (c) effects on relationships outside of the workplace. Despite their intensive knowledge about mental health and therapeutic processes, mental health professionals are still highly susceptible to vicarious trauma through constant and consistent encounters with clients' traumatic experiences.

Kanno and Giddings (2017) explained that given their empathetic engagement with traumatized victims, mental health professionals tend to experience severe emotional reactions such as terror, grief, and rage. Consequently, this may often lead to vicarious trauma; in other words, counselors develop trauma reactions secondary to exposure to clients' traumatic experiences (Trippany et al., 2004; Zaccari, 2017). It was also found that trauma work leads to changes in schemas and day-to-day routines and that these changes can be both negative and positive, implying a non-binary view of the impact of trauma work (Cohen \& Collens, 2013).

Studies focusing on vicarious trauma have already been conducted. In particular, with forensic mental health professionals (Bradford \& de Amorim Levin, 2020), Filipino first responders and masculine ideology (Agbayani et al., 2018), vicarious trauma prevention of mental health therapists (Harrison \& Westwood, 2009), vicarious resilience of mental health therapist (Hernández et al., 2010), mental health professionals with exposure to sexual trauma (Hunt, 2018), Neuro-reciprocity and vicarious trauma in psychiatrists (Isobel \& Angus-Leppan, 2018), traumatic stress in mental health professionals (Kanno \& Giddings, 2017), child welfare and child protection professionals (Molnar,

This article published by Philippine Social Science Journal (PSSJ) is licensed under a Creative Commons AttributionNoncommercial 4.0 International (CC BY-NC 4.0). You are free to share (copy and redistribute the material in any medium or format) and adapt (remix, transform, and build upon the material). Under the following terms, you must give appropriate credit, provide a link to the license, and indicate if changes were made. You may do so in any reasonable manner, but not in any way that suggests the licensor endorses you or your use. You may not use the material for commercial purposes. 
2020), mental health counselors (Sobkowiak, 2018), vicarious trauma prevention on counselors (Trippany et al., 2004), and trauma therapists (Zaccari, 2017). Almost all the studies were done internationally, and there has been limited research done regarding vicarious trauma and coping strategies of our local mental health professionals.

Given the gap in the local literature, this study consolidated the insights of mental health professionals in maintaining their professional and personal well-being while dealing with traumarelated cases. Through the utilization of the Constructivist Self-Development Theory by McCann and Pearlman (1990), the researchers identified mental health professionals' responses to clients' traumatic material. In this framework, the impact of trauma on an individual's psychological development, adaptation, and identity is marked by profound changes in the core aspects of a therapist's self or psychological foundation. In conceptualizing strategies to balance psychological needs and the development of a wellness plan, it may have a transforming effect on the personal and professional growth of trauma therapists and, in turn, enhance the healing process for client survivors (Zaccari, 2017).

\subsection{Methods}

This qualitative study scrutinized and examined the data gathered through a Narrative Inquiry research design. The utilization of qualitative research aims to give an in-depth understanding of a specific issue or gather opinions and explanations of a population, in this case, the mental health professionals with firsthand experience in dealing with vicarious trauma, toward events, beliefs, or practices (Bueno, 2016). Specifically, it is through Narrative Inquiry that enables the illumination of real people in natural settings through the 'painting' of their stories (Wang \& Geale, 2015). Knowles (2018) propounded that people's stories give access to transporting their realities and opens up one's mind to new experiences and perceptions of other people, how they think and even feel.

In the qualification, screening, and selection of conversation partners, the researchers employed purposive sampling. By definition, purposive sampling refers to a non-sampling method that can generate logically assumed population representatives (Trobia \& Lavrakas, 2011). A total of 5 conversation partners participated in this study which were represented by the following; 2 guidance counselors, 2 psychologists, and 1 psychiatrist. Further, the following inclusion criteria are being adhered to: participants must be a licensed, practicing, mental health professional (i.e., Guidance Counselor, Psychologists, or Psychiatrist); must have worked with five or more clients that experienced traumatic events (e.g., accidents, community violence, war or political violence, and domestic/ sexual or physical abuse); must have at least five years of practice as a mental health professional; and must be knowledgeable in the topic about vicarious trauma, and have experienced any of its symptoms.

In gathering data for narrative inquiry, an interview is the most appropriate instrument to be utilized. Interviews were often described as varying between the structured and the unstructured (Howitt, 2019). In this context, the researchers used an in-depth, semi-structured interview and were guided by an interview protocol matrix to gain insights from the participants' experiences. Appropriately, the researchers adopted the interview refinement protocol framework by CastilloMontoya (2016) to ensure the relevance of the questions during the interview. The said protocol is divided into four phases: (1) ensure interview questions align with research questions; (2) constructing an inquiry-based conversation; (3) receiving feedback on interview protocols, (4) piloting the interview. As the interviewer, the researchers ensured that appropriate interview decorum was observed aside from adhering to the refined interview protocol. The researchers were guided by the ethical procedures for Narrative Inquiry by Wang and Gaele (2015). The conversation partners were briefed and given an Informed Consent Form which explained the nature and rationale of the study, the benefits, obligations, possible risks, confidentiality, the extent of participation, and withdrawal terms. Further, the researchers sought their approval to video and audio record the entire interview. The procedures adhered to by the researchers in this study aimed to protect the welfare and ensure the rights of the conversation partners.

As for the interview, the conversation partners were initially oriented to be interviewed at least twice. However, due to heavy workloads brought about by a surge in the number of patients experiencing mental health concerns, the conversation partners bargained for a one-time interview which lasted on an average of 1 hour and 6 minutes for each of them. Despite this limitation, the researcher is still confident that the depth of the stories and insights gathered has reached its saturation due to repetition and duplication of ideas. 
Upon acquiring all the essential data through the interview, the researchers analyzed and explicated following the Thematic Analysis of Braun and Clarke (2006). This tool comprises six frameworks: data familiarization, generating initial codes, searching for themes, review of themes, theme definition and labeling; and report writing. As the interviews are being recorded, the data gathered from the interview were transcribed using codes. Initially, these codes were grouped into clusters, and from then, central themes were extracted.

\title{
Conversation Partners
}

To ensure the anonymity and confidentiality of the participants, the researchers used codes to alter their identities:

Conversation Partner 1 (CP1) is a married female Registered Guidance Counselor with one child working in mental health for ten years. She had various affiliations with Colleges and Universities as a Guidance Counselor and Professor. She spends her time immersed in community works and mental health advocacies.

Conversation Partner 2 (CP2) is a married female Registered Guidance Counselor with 18 years of experience and a mother of two. She handled clients seeking individual counseling, marital psychotherapy, and family interventions. CP2 is currently working as a Guidance Counselor and Professor.

Conversation Partner 3 (CP3) is a female Psychiatrist and a mother of two children. She handled different clients with traumatic experiences throughout her 29 years of practice. Additionally, CP3 had other engagements in her community and was a Professor in a distinguished medical university.

Conversation Partner 4 (CP4) is a female licensed Psychologist with 40 years of experience in the field of mental health. She is married with four children. CP4 also had significant engagements with public and private institutions catering to clients with trauma cases.

Conversation Partner 5 (CP5) is a female licensed Psychologist with over 15 years of experience. She is married with three children and spends her time volunteering in different mental health organizations in her community.

\subsection{Results and Discussion}

In this study, six central themes were lifted from the stories and insights of the five mental health professionals. These were the coping strategies that aided in maintaining their professional and personal well-being amidst constant exposure to their clients' traumatic experiences.

\section{Recognition of Experienced Vicarious Trauma Symptoms}

Mental health professionals are used to hearing stories of traumatic experiences of their clients. Consequently, they become at risk of experiencing the symptoms of vicarious trauma due to their line of work (Hunt, 2018; Isobel \& Angus-Leppan, 2018; Zaccari, 2017). It was elaborated by Zaccari (2017), who claimed that a treating therapist might also experience a mirrored reaction to a client's disclosure of their traumatic story. Thus, it may result in physical, emotional, somatic symptoms (Sobkowiak, 2018) or intrapersonal trauma (Isobel \& Angus-Leppan, 2018). In this study, the conversation partners experienced vicarious trauma symptoms, observable through their emotional response, physiological condition, behavioral reaction, and cognitive alterations.

\footnotetext{
"I was disturbed; I was having doubts if I am fit to be in this profession. I questioned myself, "Am I doing it right?", "Is this for me?" I limited myself to community outreach activities and diverted my attention to other tasks like office work. It was to divert my attention from the traumatic experience I had with my client." (CP1)
}

\begin{abstract}
"I remember that I became very vigilant, not with my clients but with my children. So, every time I felt something was wrong, I would check her body... this is because I am handling many sexually abused clients. Then I would bring her to the doctor. So imagine she has to be checked, at a very young age, for her vaginal area at the age of 2." (CP2)
\end{abstract}

"I was stressed, I got tired, I got hungry and thirsty. Nevertheless, I was glad that nothing bad happened to her (the client)." (CP4) 
"Let me recall; maybe unconsciously there was a time that I got depressed, I wanted to cry." (CP5)

Zaccari (2017) propounded that failure to recognize vicarious trauma symptoms may jeopardize the therapeutic effectiveness with their clients. Thus, the conversation partners emphasized the importance of recognition and familiarity with its symptoms. Once the symptoms were experienced and identified, the conversation partners generated their strategies to find relief. "So what I did was, I did much research for me not to be in that state again. Once you start experiencing it (vicarious trauma symptoms), you already need an intervention." (CP2)

"So we are stressed, we can get overwhelmed, we can experience the same stress responses that we find among the direct and indirect victims (of trauma). Why? Because we are human beings. Nevertheless, the difference is that we are trained to handle this. We are educated to handle this. We have to recognize signs and symptoms (of vicarious trauma) in us. We have to know how to handle it..." (CP4)

The experiences of the conversation partners with vicarious trauma have similar findings from other studies. Most of which led to a consolidation of how mental health professionals experienced vicarious trauma, its changes to the cognitive frame of reference, and their strategies of recuperating from its adverse effects (Hernández et al., 2010; Marriage \& Marriage, 2005; Zaccari, 2017). As stated in a qualitative study on Clinician Stress and Coping of mental health professionals in the Department of Psychiatry by Marriage and Marriage (2005), there has been an assumption that the essence of the concept of Vicarious Traumatization is a change in inner experience/cognitive frame of reference. All their respondents reported this, to some degree, and in different ways.

\title{
Healthy Personal and Working Relationships
}

According to the study of Harrison and Westwood (2009), when clinicians are nourished and sustained by relationships in the personal realm, they find their professional caregiving role less depleting. Thus, it is important to note that support systems such as family and friends are essential for them to cope with and adjust, especially when recalibrating emerging negative behaviors due to exposure to trauma (Lim \& Parreño, 2020). As affirmed by the conversation partners in this study, having healthy personal and working relationships has contributed to their recuperation from the symptoms of vicarious trauma.

"I am just so happy to be surrounded with the right people, who pulled me back. They encouraged me to go back to my advocacies with the notion that many people need our help." (CP1)

\begin{abstract}
"Establish good personal relationships with family, friends, and officemates. Because if your relationships are not that good, it will be traumatizing. It would be challenging for you to handle trauma cases. Avoid toxic people." (CP2)
\end{abstract}

In the work setting, McCann and Pearlman (1990) suggested that weekly case conferences and other groups for clinicians who work with traumatized clients can counter professional isolation and provide emotional support by helping to normalize and elucidate therapist reactions to client trauma. The same notion was expressed by the conversation partners, which affirmed that having a mentor and close relationships with people in the field of Mental Health has always been beneficial. "As a mental health professional, it pays to listen to the experiences of my fellow mental health professionals... I always learn something new..." (CP1)

"Do not be afraid to ask for help. Seek mentorship. I talk to Ms. Lena* and Dr. Dennis* about some of my cases. Talk with your colleagues. Solicit ideas from other colleagues in the field, kind of like a consultation." (CP2)

In view of this, mental health professionals seek human interaction and maintain harmonious relationships to receive emotional and professional guidance from the people around them. 


\title{
Fulfilling Self-Preserving Needs
}

In the Hierarchy of Needs by Abraham Maslow, the first basic need that is recurring and constantly satisfied is the physiological needs: money, food, shelter, oxygen, and sleep (Feist et al., 2013). Studies also propounded maintaining a healthy lifestyle and routine would most likely give an individual a sense of control, lessen the need to make fewer decisions, and make more time to do other things or relax (Arlinghaus \& Johnston, 2019; Kendra, 2020). In the case of therapists, losing sight of their personal needs and neglecting their self-care may lead to vicarious trauma (Zaccari, 2017). Thus, the conversation partners in this study highlighted that fulfilling their basic needs and having a structured routine is one of their coping strategies in dealing with vicarious trauma.

\begin{abstract}
"You have to take good care of yourself physically. You have to look at yourself in the mirror. It is essential because it is very painful for a mental health professional to look like one of your clients. We know very well that your appearance shows the (negative) effects. It is an indication that your sanity is still there." (CP2)
\end{abstract}

"You also need a routine before you start a session and take deep breaths... comb my hair. Inhale, exhale. I do those things before I deal with a client." (CP5)

Leisure activities are essential to the conversation partners, for it is the time to press pause on the mental and emotional demands of their jobs as mental health professionals. According to the study of Pressman et al. (2009), they found evidence of an association between having multiple types of leisure activities and psychological and physical well-being. This claim was further supported by Aksoy et al. (2017), stating that the phenomenon of leisure time is said to be helpful by giving temporary independence from life's estrangements. To make time for leisure activities, the conversation partners also expressed the importance of setting work boundaries and limits.

\footnotetext{
"Recreation. I never forget to do fun activities that will perk me up. This may include traveling with loved ones, talking with friends, limiting social media exposure, eating my favorite food, and the like. You are the most important resource, so take good care of yourself." (CP1)

"I like to sing. I also watch Netflix in my free time. For me, I try to enjoy simple pleasures even if they call it "mababaw" (superficial). I am very aware that life is too short, so we have to enjoy the little things." (CP5)

"I limit my patients. I only work half-day. So, do not push yourself against the wall and your limits because it will have significant consequences on your health. Prioritize health because if you get sick, you cannot work, you cannot function." (CP3)
}

All the responses of the conversation partners pertained to self-preservation. Self-preservation ensures the survival of oneself by making conscious decisions that would promote less harm or extinction (Cooley \& Cooley, 2017). Concerning this, Martin et al. (2020) propounded that selfpreservation practices should start early before harmful practices become set and are difficult to change. By ensuring that their bodies are in good shape and condition, they would appear pleasing to their clients and sustain long hours of counseling trauma-related cases.

\section{Regulation of Thought Processes}

Humans have a way of overcoming undesirable experiences and navigating through stressful environments. Once information is received, it is processed by the brain and released through speech, body movements, behaviors, or emotions. Through the constant exposure of detailed experiences of the mental health professionals, their minds are processing and making sense of the information they receive. Mental health professionals have an extensive understanding of psychological theories, analyze trauma-related concepts, and educate their clients with research-based options. With that much knowledge, mental health professionals are expected to regulate their thought processes better than anyone.

\footnotetext{
"The given you do not touch. You only navigate around factors that you have control with. Because the "given" you do not have control, it is already there, and you cannot touch it. So, if you focus on the 'given", it is useless energy, useless anxiety." (CP3)
} 
"Be friends on how you think and how you feel and process because we are the ones who can fine-tune ourselves. People do not own our thoughts and feelings, so it is up to us to deal with it." (CP3)

Thus, one of the conversation partners added that she used mental strategies during sessions with trauma-related clients, such as Compartmentalization, a mental strategy to comprehend opposing ideas that contradict one's principles. Mental strategies are sometimes used by individuals that may have developed different states of consciousness as a defense mechanism against painful or traumatic emotional experiences (Békés et al., 2018).

\begin{abstract}
"If there is no one available, I would have myself submit to cognitive conditioning. This is not my experience; this is the experience of my clients. Compartmentalization is very helpful for me. I need to compartmentalize." (CP2)

"After that, I tried to protect myself by using Compartmentalization so that it will not happen again. After the said method, I never had dreams about my clients again." (CP2)
\end{abstract}

Other conversation partners added that they seem to engage in internal dialogues to remind themselves of their present situations. In the study of Kross et al. (2014), it was demonstrated that small shifts in the language people use to refer to the self as they engage in this self-talk influences their ability to regulate their thoughts, feelings, and behavior.

\footnotetext{
"I realized that it is not my fault that the client took his own life. I told myself, Yes, there are still ways to improve myself handling this type of situation/clients. So next time around, I will do better. (CP1)
}

"So what I do is, I tell myself that it is normal. I remind myself that it is their problem, and I am just there to help them. By this, it gives me some boundaries. It is part of my work. Their life is not my life, and I have my own life. So that is how you deal with it." (CP4)

With these responses from mental health professionals, it is evident that they practice what they have learned throughout their years in the field. When they are in a repetitive process of recalling and recollecting the traumatic experience of their clients during counseling, it becomes a conscious effort to use mental strategies. It appeared that they handled their emotions to painful experiences of their clients with cognitive approaches. The said approaches seemed to help them understand their thoughts and gain awareness of how to recuperate from vicarious trauma or lessen its negative impact on their well-being.

\title{
Professional Competence and Due Care
}

Professional ethics provides an overview of the expectations and standards of a professional. Additionally, it also serves as a reference of behavioral rules for the counselor in carrying out their duties and responsibilities to their clients (Haryati, 2018). For the conversation partners, the code of ethics for mental health professionals was their guide in maintaining their competency and making them experts in dealing with trauma cases, thus minimizing the possible risk from the constant exposure to their clients' traumatic experiences.

"First, is to make sure you undergo training - further studies and reading. If you are in the field, you do not have the right to be lazy on reading, studying, or not training... All the knowledge you have accumulated would serve as your shield..." (CP2)

"In terms of the professional aspect, I do not stop studying. One must not stop learning because we are dealing with people. They are dynamic. We need to learn new ways to handle them." (CP5)

The conversation partners not only served as Guidance Counselors, Psychologists or Psychiatrists but are also educators to the next generations of mental health professionals. According to the study of Harrison and Westwood (2009), therapists found that diversity of 
professional roles put them into contact with a larger community, thus allowing them to feel a sense of interconnection and renewed hope.

"With my part-time teaching, I need to read to keep my mind active, to refresh my learnings. We need to develop our cognitive aspects because, in counseling, we analyze, diagnose, so it is much thinking..." (CP5)

Therefore, adherence to ethics, having various professional roles, and up-to-date training were how the mental health professionals in this study maintained their professional well-being.

\title{
Fate and Faith
}

Most conversation partners seemed to have the same recollection of becoming a mental health professional, and it was not their first choice. For them, becoming a mental health professional was somehow predetermined by some unknown forces.

\begin{abstract}
"Doing MHP started when my mentor invited me to an outreach program that they are doing. Until such time, it became my passion for serving the community. I think I was "magnetized" to be in this field... So, from one volunteer work to another, it did not stop until I decided to focus more on mental health issues." (CP1)
\end{abstract}

\begin{abstract}
"I think that was a turning point when I was not accepted in Internal Medicine (IM) because the way was paved for me to apply in Psychiatry. Moreover, I think I am meant to be in this field because I have always been a neutral person, telling me their stories..." (CP3)

"Because my mother asked me if I am sure to enroll in Fine Arts... I asked her what I would take up. She said, anything! Just not Fine Arts! So, psychology was my second choice. I am happy with it because psychology does not feel like real work. Also, I believe that this is what God wants me to be." (CP4)
\end{abstract}

Their commitment to this field may stem from their belief that this profession must be their purpose. They gave meaning to something unfathomable. Thus, the mental health professionals continued to give their services despite constantly hearing the traumatic experiences of their clients and the possible risk in this profession. They concluded that it must be fate. Their sense of commitment can be seen in their sacrifices for the profession and community they serve.

\footnotetext{
"Doing mental health activities will make you spend more time, effort, and resources. Sometimes, you make sacrifices. However, all of this will be worth it - knowing that you have shared yourself with others, helping them to feel better or be better in general." (CP1)
}

Further, the conversation partners expressed the importance of having a spiritual connection. They emphasized the importance of lifting it all to God when things do not go according to plan, whether in their clients' cases or personal endeavors. This is supported by the assumption that if a Filipino individual experiences an unexpected event in their life, whether negative or positive, it is typically attributed to the grace of God (Abad et al., 2014).

\footnotetext{
"Spiritually. There will be cases that are hard to solve, you did your best, but it would seem to fail. At the end of the day, if you surrender everything to Him and accept the fact that you are not a perfect counselor (and $\mathrm{He}$ is the perfect counselor and the first one.) We will be at peace." (CP2)
}

The responses of the mental health professionals are similar to the statements in the study of Harrison and Westwood (2009). It was found that the sense of interconnectedness with the mysterious transcendent (e.g., "this other realm ... the mystery stuff"), which is tacitly known and cannot be clearly articulated through words or otherwise apprehended, is sustaining of therapists' professional efforts and personal well-being for the reason that it reduces feelings of isolation and despair.

Mental health professionals are individuals who rely heavily on scientific studies and evidencebased data; however, when placed in a position that is detrimental or situations that are beyond the understanding of the human mind, these professionals tend to turn and seek assistance to a much higher being, God. 
As discussed, this study posited that mental health professionals deal with vicarious trauma through different coping strategies. As explained by Hunt (2018), professional therapists who work with traumatic cases identified skills and techniques to cope with trauma such as peer support and debriefings, talking to loved ones, participation in recreational activities, and engaging with a higher deity. Aside from this, time spent in research and development or any activities outside the confinements of the clinic also positively affects the well-being of the mental health professionals (Sodeke-Gregson et al., 2013). Likewise, self-care strategies were found to help them secure a harmonious life and manage stressful situations (Lewis \& King, 2019).

\subsection{Conclusion}

The conversation partners' responses provided a holistic approach to coping with vicarious trauma. All generated themes on maintaining professional and personal well-being pertained to all aspects of a person; physical (Fulfilling Self-Preserving Needs), cognitive (Regulation of Thought Processes), spiritual (Fate and Faith), occupational (Professional Competence and Due Care), and emotional (Healthy Relationships with Family and Friends). Additionally, through their recognition of vicarious trauma symptoms, they were made aware of its detrimental impact on their professional and personal well-being. Thus, they applied the aforementioned strategies, which eventually became a lifestyle.

In view of this, the results would be valuable to educational institutions and training facilities for mental health professionals specialized in trauma work. This study would aid in making courses or training programs that will equip novice mental health professionals with the skills for prevention and recuperation from vicarious trauma. Further, this would be additional literature in the field of Clinical Psychology, specifically for discussions in conducting therapy for trauma cases. Treating therapists are aware and guided with coping practices and self-preserving strategies to deal with the detrimental impact of vicarious trauma. Future researchers may opt to investigate whether factors such as numbers of years in service and personality traits would make mental health professionals more or less susceptible to vicarious trauma.

Mental health professionals are known as the healers of invisible wounds. Ironically, by working within this profession, it has also affected them in more ways than expected. To combat the detrimental effects, our mental health professionals have developed strategies to shield their personal lives and maintain their professional well-being through years of education and practice. They have developed helpful routines for themselves: they know when to stop, tend their wounds, and decompress after a day's work. However, it is still a learning process for our wounded healers. They may slip and fall and even question their abilities. Nevertheless, they will continue to recuperate and, without a doubt, tirelessly give their best to serve their community.

\section{REFERENCES}

Abad, P. J. B., Tan, M. L., Baluyot, M. M. P., Villa, A. Q., Talapian, G. L., Reyes, M. E., Suarez, R. C., Sur, A. L. D., Aldemita, V. D. R., Padilla, C. D., \& Laurino, M. Y. (2014). Cultural beliefs on disease causation in the Philippines: Challenge and implications in genetic counseling. Journal of Community Genetics, 5(4), 399-407. https://doi.org/10.1007/s12687-014-0193-1

Agbayani, B. E. M., Villaflor, P. I. A. T. M., Villaret, N. P. B., \& Hechanova, M. R. M. (2018). The role of Filipino masculine ideology on the adaptive coping, psychological well-being, and vicarious trauma of first responders. International Journal of Culture and Mental Health, 11(4), 753-762. https://doi.org/10.1080/175 42863.2018.1561736

Aksoy, Y., Çankaya, S., \& Taşmektepligil, M. Y. (2017). The effects of participating in recreational activities on quality of life and job satisfaction. Universal Journal of Educational Research, 5(6), 1051-1058. https://doi. org/10.13189/ujer.2017.050619

American Psychiatric Association (APA). (2013). Diagnostic statistical manual of mental disorders, $5^{\text {th }}$ edition. https://doi.org/10.1176/appi.books.9780890425596.744053

Arlinghaus, K. R., \& Johnston, C. A. (2019). The importance of creating habits and routines. American Journal of Lifestyle Medicine, 13(2), 142-144. https://doi.org/10.1177/1559827618818044

Bradford, J. M., \& de Amorim Levin, G. V. (2020). Vicarious trauma and PTSD in forensic mental health professionals. The Journal of the American Academy of Psychiatry and the Law, 48(3), 315-318. https://doi. org/10.29158/JAAPL.200025-20 
Békés, V., Ferstenberg, Y., \& Perry, J. (2018). Compartmentalization.

Braun, V., \& Clarke, V. (2006). Using thematic analysis in psychology. Qualitative Research in Psychology, 3(2), 77101. https://doi.org/10.1191/1478088706qp063oa

Bueno, D. C. (2016). Practical qualitative research writing. Great Books Trading.

Cafasso, J. (2016). Traumatic events. Encyclopedia of Psychology.

Castillo-Montoya, M. (2016). Preparing for interview research: The interview protocol refinement framework. Qualitative Report, 21(5).

Cohen, K., \& Collens, P. (2013). The impact of trauma work on trauma workers: A metasynthesis on vicarious trauma and vicarious posttraumatic growth. Psychological Trauma: Theory, Research, Practice, and Policy. https://doi.org/10.1037/a0030388

Cooley, S. J., \& Cooley, R. V. C. (2017). Review of self-preservation at the center of personality. Imagination, Cognition and Personality, 37(1), 95-99. https://doi.org/10.1177/0276236617712988

Feist, J., Feist, G. J., \& Robets, T. A. (2013). Theories of personality.

Harrison, R. L., \& Westwood, M. J. (2009). Preventing vicarious traumatization of mental health therapists: Identifying protective practices. Psychotherapy, 203-291. https://doi.org/10.1037/a0016081

Haryati, A. (2018). Personal integrity of Islamic counselor on professional ethics commitment. Islamic Guidance and Counseling Journal, 1(1), 11-16. https://doi.org/10.25217/igcj.v1i1.191

Hernández, P., Engstrom, D., \& Gangsei, D. (2010). Exploring the impact of trauma on therapists: Vicarious resilience and related concepts in training. Journal of Systemic Therapies. https://doi.org/10.1521/ jsyt.2010.29.1.67

Howitt, D. (2019). Introduction to qualitative research methods in psychology: Putting theory into practice. Pearson UK.

Hunt, T. (2018). Professionals' perceptions of vicarious trauma from working with victims of sexual trauma. Walden University.

Isobel, S., \& Angus-Leppan, G. (2018). Neuro-reciprocity and vicarious trauma in psychiatrists. Australasian Psychiatry, 26(4), 388-390. https://doi.org/10.1177/1039856218772223

Kanno, H., \& Giddings, M. M. (2017). Hidden trauma victims: Understanding and preventing traumatic stress in mental health professionals. Social Work in Mental Health, 15(3), 331-353. https://doi.org/10.1080/1533298 5.2016 .1220442

Kendra, C. (2020). The importance of keeping a routine.

Knowles, T. C. (2018). Stories matter: Why stories are important to our lives and culture. TCK Publishing; Tom Corson-Knowles.

Kross, E., Bruehlman-Senecal, E., Park, J., Burson, A., Dougherty, A., Shablack, H., Bremner, R., Moser, J., \& Ayduk, O. (2014). Self-talk as a regulatory mechanism: How you do it matters. Journal of Personality and Social Psychology, 106(2), 304-324. https://doi.org/10.1037/a0035173

Laidlaw, K. (2018). Vicarious trauma assessments. Precedent.

Lewis, M. L., \& King, D. M. (2019). Teaching self-care: The utilization of self-care in social work practicum to prevent compassion fatigue, burnout, and vicarious trauma. Journal of Human Behavior in the Social Environment, 29(1), 96-106. https://doi.org/10.1080/10911359.2018.1482482

Lim, M. R., \& Parreño, J. L. (2020). Compassion fatigue and coping skills of Philippine national police first responders in Bacolod City. Philippine Social Science Journal, 3(2), 127-128. https://doi.org/10.52006/main. v3i2.139

Martin, E. M., Myers, K., \& Brickman, K. (2020). Self-preservation in the workplace: The importance of well-being for social work practitioners and field supervisors. Social Work (United States), 65(1), 74-81. https://doi. org/10.1093/sw/swz040

McCann, I. L., \& Pearlman, L. A. (1990). Vicarious traumatization: A framework for understanding the psychological effects of working with victims. Journal of Traumatic Stress, 3(1), 131-149. https://doi.org/10.1007/ BF00975140

Marriage, S., \& Marriage, K. (2005). Too many sad stories: Clinician stress and coping. The Canadian Child and Adolescent Psychiatry Review, 14(4), 114.

Molnar, B. E., Meeker, S. A., Manners, K., Tieszen, L., Kalergis, K., Fine, J. E., Hallinan, S., Wolfe, J. D., \& Wells, M. K. (2020). Vicarious traumatization among child welfare and child protection professionals: A systematic review. Child Abuse and Neglect, 110 (July), 104679. https://doi.org/10.1016/j.chiabu.2020.104679

Pressman, S. D., Matthews, K. A., Cohen, S., Martire, L. M., Scheier, M., Baum, A., \& Schulz, R. (2009). Association of enjoyable leisure activities with psychological and physical well-being. Psychosomatic Medicine, 71(7), 725-732. https://doi.org/10.1097/PSY.0b013e3181ad7978

Sobkowiak, N. (2018). Mental health counselors and vicarious trauma. Counselor Education Capstones.

Sodeke-Gregson, E. A., Holttum, S., \& Billings, J. (2013). Compassion satisfaction, burnout, and secondary traumatic stress in UK therapists who work with adult trauma clients. European Journal of Psychotraumatology, 4(1), 21869.https://doi.org/10.3402/ejpt.v4i0.21869

Trippany, R. L., Kress, V. E. W., \& Wilcoxon, S. A. (2004). Preventing vicarious trauma: What counselors should know when working with trauma survivors. Journal of Counseling and Development. https://doi. org/10.1002/j.1556-6678.2004.tb00283.x 
Trobia, A., \& Lavrakas, P. (2011). Encyclopedia of survey research methods, $1^{\text {st }}$ edition. Sage Publications, Inc, 169170.

Wang, C. C., \& Geale, S. K. (2015). The power of story: Narrative inquiry as a methodology in nursing research. International Journal of Nursing Sciences, 2(2), 195-198. https://doi.org/10.1016/j.jijnss.2015.04.014

Zaccari, A. M. (2017). Vicarious trauma coping and self-care practices among trauma therapists.

\section{Correspondence:}

GRAZIELA B. SALVILLA*

grazielasalvilla@gmail.com

https://orcid.org/0000-0003-1279-834X

CHRIS JOHN S. BEDORIA

csbedoria@up.edu.ph

https://orcid.org/0000-0002-8497-7032

*Corresponding Author 